\title{
AUTHOR INDEX TO VOLUME 50
}

Author

BAILEY, DAVID H.

BAILEY, DAVID H.

Battiato, S. \&

BORHO, W.

BINI, D. \& PAN, V.

BORHO, W.

BRAMBLE, JAMES H. \& PASCIAK, JOSEPH E.

BRILLHART, JOHN, MONTGOMERY, Peter L.

\& SilvERMAN,

ROBERT D.

BUCHMANN, JOHANNES \& Williams, H. C.

BUEKENHOUT, FRANCIS \& REES, SARAH

Buell, Duncan A.

CÁBOZ, REGIS

Chandler, G. A. \& GRAHAM, I. G.

CODACCIONI,

JEAN-PIERRE

COHN, HARVEY \&

DEUTSCH, JESSE

CONN, ANDREW R., Gould, Nicholas I. M.

\& TOINT, PHILIPPE L.

Demmel, JAMES W.

DENNIS, J. E., JR. \&

LI, GUANGYE

DEUTSCH, JEsSE

ERIKSSON, KENNETH \&

JOHNSON, ClaES

FERNANDEZ-CARA, ENRIQUE \& MORENO, CARLOS

Gerasoulis, A.

Gould, Nicholas I. M.

Graham, I. G.
Title

Page

Numerical Results on the Transcendence of Constants Involving $\pi, e$, and Euler's Constant . . . . . . .

The Computation of $\pi$ to 29,360,000 Decimal Digits Using Borweins' Quartically Convergent Algorithm . . . . . .

Are There Odd Amicable Numbers Not Divisible by Three?

Efficient Algorithms for the Evaluation of the Eigenvalues of (Block) Banded Toeplitz Matrices . . . . . . . . . .

See: Battiato, S. \& Borho, W. . . . . . . . . . . .

A Preconditioning Technique for Indefinite Systems Resulting from Mixed Approximations of Elliptic Problems . . . . . . . . . . . . . . . . . . . .

Tables of Fibonacci and Lucas Factorizations $\ldots \ldots .251$

On the Infrastructure of the Principal Ideal Class of an Algebraic Number Field of Unit Rank One . . . . . . 569

The Subgroup Structure of the Mathieu Group $M_{12} \ldots \quad 595$

See: Young, JefF \& Buell, Duncan A. . . . . . .

See: LoISEAU, JeAN-Francis, Codaccioni, JeanPIERRE \& CÁBOZ, REgIS . . . . . . . . . . . . . .

Product Integration-Collocation Methods for Noncompact Integral Operator Equations . . . . . . . . . . . .

See: LoISEAU, JeAN-Francis, CodaCcioni, JeANPierRe \& CÁBOZ, REgIS . . . . . . . . . . . 501

An Explicit Modular Equation in Two Variables for $\mathbf{Q}(\sqrt{3}) \quad 557$

Testing a Class of Methods for Solving Minimization Problems with Simple Bounds on the Variables . . . . . . .

The Probability That A Numerical Analysis Problem Is Difficult ..................

A Hybrid Algorithm for Solving Sparse Nonlinear Systems of Equations . . . . . . . . . . . . . . . 155

See: Cohn, Harvey \& Deutsch, Jesse . . . . . . 557

An Adaptive Finite Element Method for Linear Elliptic Problems . . . . . . . . . . . . . . . .

Critical Point Approximation Through Exact Regularization ..................

A Fast Algorithm for the Multiplication of Generalized Hilbert Matrices with Vectors . . . . . . . . . . .

See: Conn, ANdrew R., Gould, Nicholas I. M. \& TOINT, PHILIPPE L. . . . . . . . . . . . . . .

See: Chandler, G. A. \& Graham, I. G. . . . . . . . 
Author

Grandine, Thomas A.

GRAY, HENRY L. \&

ZHANG, NIEN FAN

HALE, JACK K.,

LIN, XIAO-BIAO \&

RAUGEL, GenEVIÈVE

IWANIEC, T. \&

LUTOBORSKI, A

JOHNSON, ClAES

KANNAN, R., LENSTRA,

A. K. \& LOVÁSZ, L.

KUMAR, SUNIL

LEHMER, D. H.

LEHMER, EMMA

LENSTRA, A. K.

LI, GUANGYE

LIN, XIAO-BIAO

Llorente, Pascual \& QUER, JORDI

Llorente, Pascual \& QUER, JORDI

LOISEAU, JEAN-FRANCIS, CODACCIONI, JEAN-PIERRE \& CÁBOZ, REGIS

LOVÁSZ, L.

LUNNON, W. F.

LUTOBORSKI, A.

MONTGOMERY, PETER L.

MORENO, CARLOS

OLVER, F. W. J.

OSHER, STANLEy \&

TADMOR, EITAN

PAN, V.

PASCIAK, JOSEPH E.

QUER, JORDI

QUER, JORDI

RAUGEL, GENEVIÈVE

RAYNER, FRANCIS J.

REES, SARAH

RICHTER, GERARD R.

SCHOOF, RENÉ \&

WASHINGTON,

LAWRENCE C.
Title

Page

A Stable Evaluation of Multivariate Simplex Splines . . .

197

On a New Definition of the Fractional Difference . . . . .

513

Upper Semicontinuity of Attractors for Approximations of Semigroups and Partial Differential Equations ..... .

89

Asymptotic Expansions of Multiple Integrals of Rapidly Oscillating Functions . . . . . . . . . . .

See: ERIKsson, Kenneth \& Johnson, Claes . . . .

Polynomial Factorization and Nonrandomness of Bits of Algebraic and Some Transcendental Numbers .... .

Superconvergence of a Collocation-Type Method for Simple Turning Points of Hammerstein Equations . . . . . . .

The Sum of Like Powers of the Zeros of the Riemann Zeta Function . . . . . . . . . . . . . . . . 265

Connection Between Gaussian Periods and Cyclic Units . 535

See: Kannan, R., Lenstra, A. K. \& Lovász, L. . . 235

See: Dennis, J. E., JR. \& Li, GuAngye . . . . . . . 155

See: Hale, JaCK K., Lin, XIAO-BIaO \& RaUgel, GENEVIÈVE . . . . . . . . . . . . . 89

On the 3-Sylow Subgroup of the Class Group of Quadratic

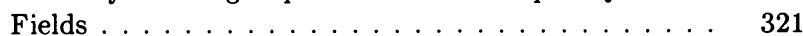

On Totally Real Cubic Fields with Discriminant $D<10^{7}$

Hyperelliptic Integrals and Multiple Hypergeometric Series 215

361

235

385

See: KAnnan, R., Lenstra, A. K. \& LovÁsz, L. . . 235 Integer Sets with Distinct Subset-Sums . . . . . . . . 297

See: IWANiec, T. \& Lutoborski, A. . . . . . . . . . 215

See: Brillhart, John, Montgomery, Peter L. \& Silverman, ROBERT D. . . . . . . . . . .

See: Fernandez-Cara, Enrique \& Moreno, CarLOS . . . . . . . . . . . . . . . . . 139

Error Bounds for Linear Recurrence Relations . . . . . . 481

On the Convergence of Difference Appoximations to Scalar Conservation Laws . . . . . . . . . . . . . . . . 19

See: BINI, D. \& PAN, V. . . . . . . . . . . . . 431

See: Bramble, James H. \& Pasciak, Joseph E. . . 1

See: Llorente, Pascual \& QUeR, JoRdi . . . . . . 321

See: LlORENTE, PASCUAL \& QUeR, JoRdi . . . . . . 581

See: HALE, JACK K., LiN, XIAO-BIAO \& RAUGEL, GENEVIÈVE . . . . . . . . . . . . . . 89

Weak Uniform Distribution for Divisor Functions. I . . 335

See: Buekenhout, Francis \& ReEs, SARAH . . . . 595

An Optimal-Order Error Estimate for the Discontinuous Galerkin Method . . . . . . . . . . . . 75

Quintic Polynomials and Real Cyclotomic Fields with Large Class Numbers . . . . . . . . . . . . . . . . 543 
SEAH, E. \& STINSON, D. R. On the Enumeration of One-Factorizations of Complete

SILVERMAN, ROBERT D.

STEPHENS, A. J. \&

WILliams, H. C.

STINSON, D. R.

TADMOR, EITAN

TEZUKA, SHU

TOINT, PHILIPPE L.

VILA, J. P.

WASHINGTON,

LAWRENCE C.

WEISER, ALAN \&

ZARANTONELLO,

SERGIO E.

WILLIAMS, H. C.

Williams, H. C.

WONG, R.

YOUNG, JEFF \& BUEll, DUNCAN A.

ZARANTONELLO, SERGIO E.

ZHANG, NIEN FAN

ZULEHNER, WALTER Graphs Containing Prescribed Automorphism Groups

See: Brillhart, John, MONTGOMERY, Peter L. \& SILVERMAN, ROBERT D. . . . . . . . . . . .

607

251

Some Computational Results on a Problem Concerning Powerful Numbers . . . . . . . . . . . . . . . . . . 619

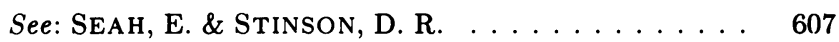

See: OSher, Stanley \& TAdMOR, Eitan . . . . . . 19

On Optimal GFSR Pseudorandom Number Generators . 531

See: CONN, ANDREW R., GOULD, Nicholas I. M. \& TOINT, PHILIPPE L. . . . . . . . . . . . . . . 399

High-Order Schemes and Entropy Condition for Nonlinear Hyperbolic Systems of Conservation Laws . . . . . . 53

See: SCHOOF, RENÉ \& WaShington, LAWRENCE C. 543

A Note on Piecewise Linear and Multilinear Table Interpolation in Many Dimensions . . . . . . . . . . . . . 189

See: Buchmann, Johannes \& Williams, H. C. . . 569

See: Stephens, A. J. \& Williams, H. C. . . . . . 619

Asymptotic Expansion of $\int_{0}^{\pi / 2} J_{\nu}^{2}(\lambda \cos \theta) d \theta \ldots \ldots .229$

The Twentieth Fermat Number is Composite . . . . . 261

See: Weiser, Alan \& Zarantonello, Sergio E. . $\quad 189$

See: Gray, Henry L. \& Zhang, Nien fan . . . . . 513

A Simple Homotopy Method for Determining All Isolated Solutions to Polynomial Systems . . . . . . . . . . . . 